\subsection{1.-17.01.2004}

Colmar

Frankreich

Deutschland

\subsubsection{4 \\ Rotenburg}

20. Jahrestagung der Oberrheinischen Arbeitsgemeinschaft Onkologie OAO

\section{Rotenburger Mamma-Symposium}

\subsection{2.-15.02.2004}

Düsseldorf

Deutschland

\subsection{2.-14.02.2004}

St. Gallen

Schweiz

\subsection{2.-21.02.2004 \\ St. Gallen}

Schweiz

\subsection{2.-21.02.2004}

Tübingen

Deutschland

\subsection{2.-28.02.2004}

Hamburg

Deutschland

\subsection{2.-01.03.2004}

Berlin

Deutschland

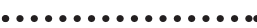

25.03.-27.03.2004

Frankfurt

Deutschland

\section{Teaching Course "Clinical Oncology 2004/05"}

3. Int. Conference: Controversies in Tumor Prevention and Genetics 2004

\section{4. Ärzte-Fortbildungskurs in Klinischer Onkologie}

10. AGO-Winterkurs

Multimodale Therapiekonzepte in der gastrointestinalen Onkologie
48. Jahrestagung der Deutschen Gesellschaft für Thrombose- und Hämostaseforschung

\section{Deutscher Krebskongress}

\section{Der deutsche Schmerztag 2004}

26.03.-27.03.2004

Erlangen

Deutschland
Knochen- und Weichteilsarkome im Erwachsenen- und Kindesalter
Auskunft: Dr. med. Bruno Audhuy

Oncohématologie, Hôpital PASTEUR

39 avenue de la Liberté

F-68024 Colmar

Tel.: +33 389804102 , Fax: +33389804343

Auskunft: Prof. Dr. med. Harald Meden

Chefarzt der Frauenklinik

des Diakoniekrankenhauses Rotenburg

Elise-Averdieck-Str. 17

D-27356 Rotenburg

Tel. +49 4261 77-2300, Fax -2144

E-mail gynsekre@diako-online.de

www.diako-online.de/frauenklinik

Auskunft: S. Roth MD

Strahlentherapeutische Universitätsklinik

Moorenstr. 5

D-40225 Düsseldorf

Tel. +49 211 811-8989, Fax -8051

Auskunft: Conference Secretariat "TUP-04"

Frau Beatrice Nair

Rorschacherstr. 150

CH-9006 St. Gallen

Tel. +41 71-243 0032, Fax -245 6805

E-mailinfo@oncoconferences.ch

www.oncoconferences.ch

Auskunft: Kongress-Sekretariat / ESO-D

Doris Hoevel

Rorschacherstr. 150

CH-9006 St. Gallen

Tel. +41 71-243 0032, Fax -245 6805

E-mail eso-d@sg.zetup.ch

www.kssg/kongresse.ch

Auskunft: Regine Zennss-Reimann M.A.

Kongress-Sekretariat, Medizinische Klinik I

Otfried-Müller-Str. 10

D-72076 Tübingen

Tel. +49 7071 29-82098, Fax -5754

E-mail regine.zennss-reimann@med.uni-tuebingen.de www.gastro-1.de

Auskunft: Congress Partner GmbH

Markgrafenstr. 56,

D-10117 Berlin

Tel. +49 30 20459-0, Fax -50

www.gth-online.org

Auskunft: Kongress- und Kulturmanagement GmbH Postfach 3664

D-99407 Weimar

E-mail info@krebskongress2004.de

www.krebskongress2003.de

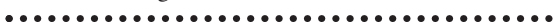

Auskunft: Interplan Congress Meeting \&

Event Management

Albert-Rosshaupter Str. 65

D-81369 München

Tel./Fax +49 $89548234-13$

E-mail schmerztag@i-plan.de

www.stk-ev.de

Auskunft: European School of Oncology

Deutschsprachiges Programm (ESO-D)

Frau Doris Hoevel

Rorschacherstr. 150

CH-9006 St. Gallen

Tel. +41 71-243 0032, Fax -245 6805

E-mail eso-d@sg.zetup.ch

\title{
KARGER
}


02.04.-03.04.2004

Baltimore

USA

\subsection{4.-17.04.2004 \\ Wien}

Österreich

\section{[. . . . . . . . . . \\ Wilsede}

Deutschland

\section{•...............}

13.05.-15.05.2004

Warth

Deutschland

\section{[.. . . . . . . . . . .}

New Orleans

USA

\section{................ \\ 10.06.-13.06.2004}

Erfurt

Deutschland

\subsection{6.-13.06.2004}

Genf

Schweiz

\subsection{6.-19.06.2004}

Dresden

Deutschland

\subsection{6.-26.06.2004 \\ Wilsede}

Deutschland
Current Concepts in the Mulitdisciplinary Management of Breast Cancer

Frühjahrstagung 2004 der Österreichischen Gesellschaft für Hämatologie und Onkologie und der Arbeitsgemeinschaft hämato-onkologischer Pflegepersonen in Österreich

\section{Wilsede-Schule für Onkologie und Hämatologie: Myelodysplastisches} Syndrom - Colorectale Karzinome - Klinische Pharmakologie

\section{Int. Seminar: Palliativ-Betreuung von Tumorkranken}

\section{Asco 40th Annual Meeting}

\section{A}

10. Jahreskongress der Deutschen Gesellschaft für Radioonkologie

\section{9.}

9. Congress of the European Hematology Association

Update Hämatologie/Onkologie 2004

Wilsede-Schule für Onkologie und Hämatologie: Palliativmedizin - Karzinome des oberen Gastrointestinaltraktes und Pankreas - Gerinnungsprobleme in der Onkologie

\section{Update Hämatologie/Onkologie 2004}

Auskunft: Program Coordinator

Johns Hopkins University

Turner 20/720 Rutland Avenue

USA-Baltimore, Maryland 21205

Tel. +1 410-955 2959, Fax -614 8613

E-mail cmenet@jhmi.edu

www.hopkinscme.org/cme

Auskunft: Mondial Congress

Faulmanngasse 4

A-1040 Wien

Tel. +43 1-588 040, Fax -5869185

E-mail congress@mondial.at

Auskunft: Frau Klara Schlunk

Klinikum Oldenburg

Klinik für Innere Medizin II

Dr. Eden Str. 10

D-26133 Oldenburg

Tel. +49 441 403-2611, Fax -2654

E-mail illiger@klinikum-oldenburg.de

Auskunft: Kongress-Sekretariat / ESO-D

Doris Hoevel

Rorschacherstr. 150

CH-9006 St. Gallen

Tel. +41 71-243 0032, Fax -245 6805

E-mail eso-d@sg.zetup.ch

www.kssg/kongresse.ch

Auskunft: ASCO

1900 Duke Street, Suite 200

Alexandria, VA USA

Tel. +1 703 299-0150, Fax -1044

E-mail asco@asco.org

Auskunft: Kongressgesellschaft der Degro mbH

Warnstedtstr. $70 \mathrm{c}$

D-22525 Hamburg

Tel. +49 40-54768507, Fax -52014846

E-mail Degro-Thoel@t-online.de

Auskunft: Eurocongres Conference Management

Jan van Goyenkade 11

NL-1075 HP Amsterdam

Tel. +31 20-679 3411, Fax -673 7306

E-mail eha2004@eurocongres.com

Auskunft: Prof. Dr. S. Petrasch

Klinikum Duisburg

Zu den Rehwiesen 9

D-47055 Duisburg

Tel. +49 203 73323-00, Fax -02

E-mail s.petrasch@t-online.de

www.onko-update.de

Auskunft: Frau Klara Schlunk

Klinikum Oldenburg

Klinik für Innere Medizin II

Dr. Eden Str. 10

D-26133 Oldenburg

Tel. +49 441 403-2611, Fax -2654

E-mail illiger@klinikum-oldenburg.de

•..............................

Auskunft: Prof. Dr. S. Petrasch

Klinikum Duisburg

Zu den Rehwiesen 9

D-47055 Duisburg

Tel. +49 203 73323-00, Fax -02

E-mail s.petrasch@t-online.de

www.onko-update.de 
25.06.-26.06.2004

Ontario

Kanada

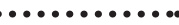

02.07.-03.07.2004

Stuttgart

Deutschland

-...............

22.07.-24.07.2004

Zürich

Schweiz

\subsection{9.-04.09.2004}

Freiburg

Deutschland

\subsection{9.-18.09.2004 \\ Stuttgart}

Deutschland

\subsection{9.-02.10.2004 \\ Berlin}

Deutschland

\subsection{0.-06.10.2004 \\ Innsbruck}

Österreich

\section{................}

21.10.-23.10.2004

Freiburg

Deutschland

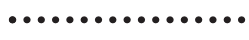

\subsection{0.-02.11.2004}

Wien

Österreich

\section{[.................}

26.01.-29.01.2005

St. Gallen

Schweiz
8. International Conference on Long-Term Complications of Treatment of Children and Adolescents for Cancer

\section{Update Hämatologie/Onkologie 2004}

International Skin Cancer Conference

24. Jahrestagung der Deutschen Gesellschaft für Senologie

12. Internationaler Kongress für Gesundheitsberufe in Diagnostik, Therapie und Akutversorgung (bisher Funktionsdienstekongress)

Gastrointestinale Tumoren + Update Mammakarzinom 2004

Gemeinsame Jahrestagung der Deutschen, Österreichischen und Schweizerischen Gesellschaft für Hämatologie und Onkologie

\section{International Freiburg Symposium on Molecular Hematology}

\section{ESMO Congress}

9. International Conference on Primary Therapy of Early Breast Cancer
Auskunft: Daniel Green, MD, Pediatrics

Roswell Park Cancer Institute

Elm \& Carlton Streets

Buffalo, NY 14263

Tel. +1 716 845-2334, Fax -8003

E-mail daniel.green@roswellpark.org

Auskunft: Prof. Dr. S. Petrasch

Klinikum Duisburg

Zu den Rehwiesen 9

D-47055 Duisburg

Tel. +49 203 73323-00, Fax -02

E-mail s.petrasch@t-online.de

www.onko-update.de

Auskunft: Reinhard Dummer, M.D.

Dept. of Dermatology

University Hospital Zürich

Gloriastr. 31, CH-8091 Zürich

Tel. +41 1 255-8837, Fax -4403

E-mail nicole.brunner@usz.ch

www.skincancer.ch

Auskunft: CTW - Congress Organisation Thomas

Wiese $\mathrm{GmbH}$

Goßlerstr. 30

D-12161 Berlin

Tel. +49 30 85-996216, Fax -079826

E-mail senologie@ctw-congress.de

..............................

Auskunft: MCN Medizinische Congressorganisation

Nürnberg AG

Zerzabelshofstr. 29

D-90478 Nürnberg

Tel. +49 911 39316-17, Fax -20

E-maildbfk@mcnag.info

www.mon-nuernberg.de

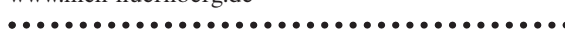

Auskunft: European School of Oncology -

Deutschsprachiges Programm (ESO-D)

Frau Doris Hoevel

Rorschacherstr. 150

CH-9006 St. Gallen

Tel. +41 71-243 0032, Fax -245 6805

E-mail eso-d@sg.zetup.ch

Auskunft: Mondial Congress

Faulmanngasse 4

A-1040 Wien

Tel. +43 1-588 040, Fax -586 9185

E-mail dgho-ögho2004@mondial.at

Auskunft: Prof. H. Veelken

Medizinische Klinik I

Universitätsklinikum Freiburg

Hugstetter Str. 55

D-79106 Freiburg

Tel. +49 761 270-3462 / 3530, Fax -3233

E-mail veelken@mm11.ukl.uni-freiburg.de

Auskunft: ESMO

Congress Secretariat

Via La Santa 7

CH-6962 Viganello-Lugano

Tel. +41 91 950-0781, Fax -0782

E-mail alessia@esmo.org, chatrina@esmo.org

www.esmo.org

.................................

Auskunft: St. Gallen Oncology Conferences

Frau Beatrice Nair

Rorschacherstr. 150, POB

CH-9006 St. Gallen

Tel. +41 71-243 0032, Fax -245 6805

E-mailinfo@oncoconferences.ch

www.oncoconferences.ch 